\title{
A synthetic biosensor to detect peroxisomal acetyl-CoA concentration for compartmentalized metabolic engineering
}

\author{
Herbert M Huttanus ${ }^{1}$, Ryan S Senger ${ }^{\text {Corresp. } 1,2}$ \\ ${ }^{1}$ Department of Biological Systems Engineering, Virginia Polytechnic Institute and State University (Virginia Tech), Blacksburg, Virginia, United States \\ 2 Department of Chemical Engineering, Virginia Polytechnic Institute and State University (Virginia Tech), Blacksburg, Virginia, United States \\ Corresponding Author: Ryan S Senger \\ Email address: senger@vt.edu
}

Background. Sub-cellular compartmentalization is used by cells to create favorable microenvironments for various metabolic reactions. These compartments concentrate enzymes, separate competing metabolic reactions, and isolate toxic intermediates. Such advantages have been recently harnessed by metabolic engineers to improve the production of various high-value chemicals via compartmentalized metabolic engineering. However, measuring sub-cellular concentrations of key metabolites represents a grand challenge for compartmentalized metabolic engineering.

Methods. To this end, we developed a synthetic biosensor to measure a key metabolite, acetyl-CoA, in a representative compartment of yeast, the peroxisome. This synthetic biosensor uses highly efficient enzyme re-localization via PTS1 signal peptides to construct a metabolic pathway in the peroxisome which converts acetyl-CoA to polyhydroxybutyrate (PHB) via three enzymes. The PHB is then quantified by HPLC.

Results. The biosensor demonstrated the difference in relative peroxisomal acetyl-CoA availability under various culture conditions and was also applied to screening a library of single knockout yeast mutants. The screening identified several mutants with drastically reduced peroxisomal acetyl-CoA and one with potentially increased levels. We expect our synthetic biosensors can be widely used to investigate subcellular metabolism and facilitate the "design-build-test" cycle of compartmentalized metabolic engineering. 


\section{A Synthetic Biosensor to Detect Peroxisomal Acetyl-CoA}

2 Concentration for Compartmentalized Metabolic

3 Engineering

4

5 Herbert M. Huttanus ${ }^{1}$, Ryan S. Senger ${ }^{1,2}$

6

$7 \quad{ }^{1}$ Department of Biological Systems Engineering, Virginia Polytechnic Institute and State

8 University (Virginia Tech), Blacksburg, VA, USA

$9{ }^{2}$ Department of Chemical Engineering, Virginia Polytechnic Institute and State University

10 (Virginia Tech), Blacksburg, VA, USA

11

Corresponding Author:

13 Ryan S. Senger ${ }^{1,2}$

14 Department of Biological Systems Engineering, Virginia Tech, 1230 Washington St., 301C

15 HABB1, Blacksburg, VA, 24061, USA

16 Email address: senger@vt.edu

Keywords: acetyl-CoA, biosensor, compartmentalization, peroxisome, quantification

Abbreviations: CoA, coenzyme A; DCW, dry cell weight; HPLC, high performance liquid 


\section{Abstract}

24

25 Background. Sub-cellular compartmentalization is used by cells to create favorable

26 microenvironments for various metabolic reactions. These compartments concentrate enzymes, 27 separate competing metabolic reactions, and isolate toxic intermediates. Such advantages have 28 been recently harnessed by metabolic engineers to improve the production of various high-value 29 chemicals via compartmentalized metabolic engineering. However, measuring sub-cellular 30 concentrations of key metabolites represents a grand challenge for compartmentalized metabolic 31 engineering.

32 Methods. To this end, we developed a synthetic biosensor to measure a key metabolite, acetyl$33 \mathrm{CoA}$, in a representative compartment of yeast, the peroxisome. This synthetic biosensor uses

34 highly efficient enzyme re-localization via PTS1 signal peptides to construct a metabolic pathway 35 in the peroxisome, which converts acetyl-CoA to polyhydroxybutyrate (PHB) via three enzymes. 36 The PHB is then quantified by HPLC.

37 Results. The biosensor demonstrated the difference in relative peroxisomal acetyl-CoA 38 availability under various culture conditions and was also applied to screening a library of single 39 knockout yeast mutants. The screening identified several mutants with drastically reduced 40 peroxisomal acetyl-CoA and one with potentially increased levels. We expect our synthetic 41 biosensors can be widely used to investigate sub-cellular metabolism and facilitate the "design42 build-test” cycle of compartmentalized metabolic engineering. 


\section{Introduction}

44

45

46

47

48

49

50

51

52

53

54

55

56

57

58

59

60

61

62

63

64

65

66

67

68

69

70

71

72

73

74

75

76

77

78

79

80

81

82

83

84
Sub-cellular compartmentalization is used by all eukaryotes and some prokaryotes as a means to create favorable micro-environments for various metabolic reactions. These compartments concentrate enzymes and substrates(Choi \& Montemagno, 2006), increasing the rate of reaction. Compartmentalization also helps to keep incompatible pathways separate(Zecchin et al., 2015) and can provide a safe area for toxic intermediates. Recently, metabolic engineers have explored the concept of pathway compartmentalization to enhance the performance of metabolic pathways(Avalos, Fink \& Stephanopoulos, 2013; Zecchin et al., 2015; Zhou et al., 2016).

One challenge to the continued progress of compartmentalized metabolic engineering is the lack of tools for measuring local metabolite concentrations at a sub-cellular scale. There are many proprietary colorimetric and fluorescent assays (such as glucose peroxidase assays(Ngo \& Lenhoff, 1980) or enzymatic acetyl-CoA assays(Fritz et al., 2013)) but these in vitro methods often require cell lysis. Cell fractionation involves separating cell compartments of interest beforehand, but contamination with similarly sized organelles is possible following differential or density gradient centrifugation(Kikuchi et al., 2004). In vivo biosensors are another popular method for screening the response of metabolites to various perturbations. These techniques often involve the use of transcription factors that recognize the analyte and activate bioluminescence or fluorescence reporter genes(Michener et al., 2012; Mustafi et al., 2012; Brognaux et al., 2013; Li \& Yu, 2015; Mahr et al., 2016; Skjoedt et al., 2016). This, generally, means that the detection of the analyte largely occurs in the cytoplasm or nucleus.

To provide a tool for measuring metabolite availability in sub-cellular compartments, we propose using a localized synthetic biosensor as a form of metabolic assay. In this method, heterologous enzymes are expressed with localization tags to produce some reporter compound only in the compartment of interest. With sufficiently high levels of enzyme expression, the production of the reporter compound would be rate-limited by the substrate concentration, allowing for relative quantification of the substrate. Enzymes should be selected that use either no substrates besides the metabolite of interest or that use cofactors that are much more available than the metabolite of interest such that the cofactors do not become limiting. The reporter compound can then be extracted from the cell and quantified. Herein, we showcase the use of one such synthetic biosensor to measure acetyl-CoA in the peroxisomes of the yeast Saccharomyces cerevisiae. Acetyl-CoA is a core metabolite whose availability has implications in many important metabolic pathways. Measuring total, cellular acetyl-CoA levels is a simple matter using commercially available enzymatic assay kits(Jose \& Suraishkumar, 2016), but measuring acetyl-CoA in organelles is more challenging since conventional assays would require the organelles to first be isolated by cell fractionation. Polyhydroxybutyrate (PHB) production can also be used as an indirect marker of acetyl-CoA availability. Only three enzymes are needed for the conversion of acetyl-CoA into PHB(Wang \& Yu, 2007) and they can be efficiently localized to the peroxisome by inclusion of C-terminal PTS1 peptide tags(Kim \& Hettema, 2015). Acetyl-CoA does not readily cross membranes(Chen, Siewers \& Nielsen, 2012), so the peroxisome-localized enzymes 
85 should only have access to the peroxisomal pool of acetyl-CoA (Fig. 1). In this paper, a localized

86 enzymatic assay and HPLC analysis of the cellular PHB content were used to qualify the

87 response of acetyl-CoA in the peroxisome to several culturing conditions and gene knockout

88 perturbations.

89

90 Materials and methods

91

92

\section{Media and strains}

93

94

Minimal media was prepared as previously reported(Blank \& Sauer, 2004; Guo et al., 2016) with a glucose concentration of $0.5 \% \mathrm{w} / \mathrm{v}$. Yeast nitrogenous media YN contains $0.67 \% \mathrm{w} / \mathrm{v}$ yeast nitro base, $0.1 \%$ yeast extract and $0.5 \%$ glucose. Synthetic complete media contained $0.17 \%$ yeast nitro base, $0.5 \%$ ammonium sulfate, $0.5 \%$ glucose and $1 \mathrm{x}$ CSM Ura supplement from MP biomedicals. Oleic acid was added to the media in some cases to enhance acetyl-CoA availability in the peroxisome through beta oxidation. All cultures were incubated at $30^{\circ} \mathrm{C}$ in a shaking incubator. The experiment testing for the effect of media composition used $10 \mathrm{~mL}$ cultures each and the mutant screening cultures were $3 \mathrm{~mL}$ cultures each from which only $2 \mathrm{~mL}$ was harvested at the end of culturing. The growth/production curve experiments used $20 \mathrm{~mL}$ cultures from which $2 \mathrm{~mL}$ were drawn at each time point. All of the homologous recombinations were performed in S. cerevisiae Invitrogen strain INVSc1. Plasmids were transferred to E. coli strain Top10 for verification and long-term storage. All cultures used to collect and measure PHB were performed in S. cerevisiae strain BY4741 or BY4741-derived single gene knockouts.

\section{Cloning}

111

112

113

The major plasmids and strains are shown in Table 1. Other strains and plasmids are detailed in Supplementary Methods S1. The three pha genes were codon-optimized for yeast and kindly provided by Jens Nielsen(Kocharin et al., 2012). Construction of the plasmids pPHBc and pPHBp was accomplished by an established, yeast homologous recombination-based method, DNA assembler(Shao \& Zhao, 2009). Briefly, DNA fragments were amplified by PCR using primers that add homologous overlap between adjacent fragments. The fragments were then cotransformed into $S$. cerevisiae along with a linearized backbone to assemble elements in a single step(Shao, Luo \& Zhao, 2011). Gene fragments used to construct plasmid pPHBp used modified primers for the addition of ePTS1 localization tags(DeLoache, Russ \& Dueber, 2016; Liu \&

\section{HPLC analysis}

122

Culture samples of $10 \mathrm{~mL}$ were centrifuged at $4000 \mathrm{rpm}$ for five minutes, and rinsed twice with

$12410 \mathrm{~mL}$ of $\mathrm{ddH}_{2} 0$. Pellets were then dried for 48 hours at $70^{\circ} \mathrm{C}$. PHB is insoluble, so it is regularly

125 converted to crotonic acid monomers by sulfuric acid digestion(Karr, Waters \& Emerich, 1983).

126 The pulverized dry pellets (or thin films for smaller samples) were digested with $100 \mu \mathrm{L}$ of 
127

128

129

130

131

132

\section{Results}

134

135

136

137

138

139

140

141

142

143

144

145

146

147

148

149

150

151

152

153

154

155

156

157

158

159

160

161

162

163

164

165

166

167

concentrated $\mathrm{H}_{2} \mathrm{SO}_{4}$ per $\mathrm{mL}$ of the pre-dried sample. Acid digests were carried out at $95^{\circ} \mathrm{C}$ for 1 hour. The digest was diluted with $400 \mu \mathrm{L}$ of $\mathrm{ddH}_{2} 0$ for every $100 \mu \mathrm{L}$ of acid used. The carbonified cell debris was removed by centrifugation at 13,000 rpm for $10 \mathrm{~min}$. These samples were analyzed by HPLC with an Aminex HPX-87H ion exclusion column with a temperature of $60^{\circ} \mathrm{C}$ and a flow rate of $0.6 \mathrm{~mL}$ min- 1 and with $5 \mathrm{mM} \mathrm{H}_{2} \mathrm{SO}_{4}$ as the mobile phase.

\section{Dependence on fatty acids}

To test the viability of the localized synthetic biosensor, a proof-of-concept study was performed in which yeast cells with PHB-producing genes (with or without peroxisomal localization) were analyzed to compare acetyl-CoA levels in the cytosol and peroxisome. PHB production was achieved by expressing three enzymes from Ralstonia eutropha H16. The first enzyme, acetylCoA acetyltransferase [EC 2.3.1.9], consumes two acetyl-CoA to produce acetoacetyl-CoA. The next enzyme, NADPH-dependent acetoacetyl-CoA reductase [EC 1.1.1.36] reduces the acetoacetyl-CoA in order to generate 3-hydroxybutyric-CoA monomers. The monomers were then polymerized by the final enzyme, PHB synthase [EC 2.3.1.-](Wang \& Yu, 2007). These three enzymes are encoded by the genes $p h a A$, phaB and phaC respectively. The genes were previously codon-optimized for use in yeast cells(Kocharin et al., 2012). All three genes were combined in each of two plasmids. Plasmid pPHBc contained the codon-optimized genes without peroxisomal localization tags and thus the enzymes localize in the cytoplasm. Plasmid pPHBp contains the same three enzymes but with peroxisomal localization tags designed for rapid and efficient import(DeLoache, Russ \& Dueber, 2016) which were added by homologous recombination(Joska et al., 2014). We chose PHB as our reporter compound because of two considerations. First, PHB is detected relatively easily and is non-native to yeast cells. Second, the PHB pathway is relatively short with only three enzymes, which reduces the risk of metabolic burden. One limitation of this biosensor is that the PHB production pathway also uses NADPH as a cofactor allowing for the possibility that NADPH will become the limiting reagent at sufficiently high acetyl-CoA levels.

To show that peroxisomal acetyl-CoA could be used for PHB production, we cultured the peroxisomal and cytosolic variants of the PHB producing strains (phbP and phbC respectively) in various media with various external concentrations of oleic acid for 48 hours. Three media were used: synthetic complete yeast media (SC), minimal media, and yeast nitro base (YN) media. Peroxisomal levels of acetyl-CoA were enhanced by the addition of oleic acid which, as a fatty acid, is broken down through beta oxidation in the yeast peroxisome to yield acetyl-CoA(Chen, Siewers \& Nielsen, 2012). Oleic acid content ranging from $0 \%, 0.2 \%$ and $0.5 \% \mathrm{w} / \mathrm{v}$ was added into each of the media. The cytosolic pha genes were able to produce similar levels of PHB with any of the media and at all of the oleic acid concentrations present (Fig. 2A). No PHB was produced by the peroxisomal strain phbP when oleic acid was absent from the media (Fig. 2B).

PeerJ reviewing PDF | (2019:10:41850:1:1:NEW 3 Jul 2020) 
168 Also, we found that with the increase of oleic acid concentration in the YN media, the PHB

169 production also increased.

170

171 Growth and production curves

172

173 Both strains were then cultured in YN media with 0.5\% oleic acid and sampled periodically until

174 PHB production plateaued (Fig. 3). Strain phbC achieved a maximal PHB level of around $30 \mathrm{mg} /$

175 g DCW within only 24 hours, which was the same amount of time it took to achieve a maximum

176 cell density ( $\sim$ O.D.). Strain phbP reached the same cell density in those 24 hours, but only

177 started producing PHB after that point. PHB production in the phbP strain reached a maximum of

178 about $7 \mathrm{mg} / \mathrm{g}$ DCW after five days.

179

\section{Knockout screening}

181

We demonstrated the application of our compartmentalized biosensor by screening several mutants for altered levels of peroxisomal acetyl-CoA. In brief, thirteen yeast single knockout mutants were selected and transformed with pPHBp. Three of the genes, adr1, aat2 and ino1 are transcription factors that promote fatty acid synthesis or fatty acid utilization. Three of the genes, rpd3, opi1 and sin3 are transcription factors that repress fatty acid synthesis. The genes pot1 and pox1 were selected as integral enzymes required for beta-oxidation. Genes pex 5 and pex7 are peroxisome transport proteins responsible for import of peroxisome-specific proteins. The gene inp2 was selected for its role in peroxisomal inheritance and the two genes slt2 and atg36 were selected for their role in pexophagy. The peroxisomal PHB enzymes were expressed in all thirteen knockout mutants and the wild-type yeast for five days in YN media at varied concentrations of oleic acid (Fig. 4). Beta oxidation gene knockouts $\Delta p o t 1$, and $\Delta p o x 1$, as well as peroxisomal transport protein knockouts $\Delta p e x 5$ and $\Delta p e x 7$ exhibited dramatic decreases in PHB production. Knockout strain $\triangle a a t 2$ showed relatively little change relative to the wild type while $\Delta a d r 1$ exhibited decreased PHB production(Fig. 4a). None of the three fatty acid synthesis repressor knockouts $(\Delta r p d 3, \Delta \sin 3$ and $\Delta$ opi1) showed a strong increase in PHB production but $\Delta \sin 3$ and $\Delta$ opi1 exhibited decreased production of PHB at $0.5 \%$ oleic acid relative to the wild type.

199

200

203 Evidence for peroxisomal localization of the PHB producing enzymes is first demonstrated in 204 Fig. 2. The cytosolic variant of the PHB pathway was not significantly affected by external oleic 205 acid concentrations because acetyl-CoA in the cytosol was mostly derived from glucose. The 206 peroxisomal variant of the PHB pathway, however, demonstrated a strong correlation between 207 oleic acid content and PHB production, because acetyl-CoA in the peroxisome is mostly derived 208 from beta-oxidation of fatty acids such as oleic acid(Chen, Siewers \& Nielsen, 2012). There was 209 also no detectable PHB produced from the peroxisomal pathway in Fig. 2 when oleic acid was 
210 absent, suggesting minimal leakage of cytosolic acetyl-CoA into the peroxisome. Therefore, it is 211 appropriate to use PHB production from the compartmentalized biosensor to indicate relative

212 peroxisomal acetyl-CoA availability.

213

214 Even with similar levels of glucose and oleic acid, the peroxisomal variant strain, phbP did not 215 produce as much PHB as the cytosolic variant, phbC, within 48 hours (Fig. 2), so it was 216 hypothesized that two days of incubation was not long enough for the yeast cells to fully switch 217 from glucose utilization to fatty acid utilization(Gurvitz \& Rottensteiner, 2006). Growth and 218 production curves, shown in Fig. 3 demonstrate that PHB production in the phbP strain started 219 only after 24 hours, which is the same amount of time it took to reach stationary phase. This 220 suggests that the yeast cells used glucose for cellular growth until it was depleted, then switched 221 to beta-oxidation of oleic acid. However, the strain phbP was never able to generate as much 222 PHB as the cytosolic control, phbC, even when given similar quantities of oleic acid and glucose 223 and when given ample time to utilize oleic acid.

224

225 The compartmentalized biosensor was then applied to screening several single-gene knockouts 226 for altered acetyl-CoA availability in the peroxisome. As expected, $\Delta p o t 1, \Delta p o x 1, \Delta p e x 5$ and $227 \Delta p e x 7$ all exhibited dramatic decreases in peroxisomal PHB production as those genes are 228 necessary for beta oxidation. Similarly, $\Delta a d r 1$ reduced PHB production because of its role in 229 activating fatty acid utilization. It is puzzling, however, that the $\Delta$ pex 5 strain did not exhibit high 230 levels of cytosolic PHB production. Since pex5p (the transport protein coded for by pex5) is 231 needed for peroxisomal localization of PTS1-tagged proteins, it was expected that the biosensor 232 enzymes would accumulate in the cytosol as has been observed for PTS1 tagged yellow 233 fluorescent protein(DeLoache, Russ \& Dueber, 2016). However, the pex5 knockouts in this study 234 did not perform similarly to the strains using enzymes without PTS1 tags. The reason for this is 235 still unclear.

236

237 Other knockouts were predicted to increase peroxisomal acetyl-CoA. Fatty acids generated by the 238 cell could be used to supplement the exogenous source of oleic acid for beta-oxidation. As 239 repressors of fatty acid synthesis, $\Delta r p d 3, \Delta \sin 3$ and $\Delta o p i 1$ had potential to increase PHB levels by 240 providing additional fatty acids from the cytosol to be digested in the peroxisome. However, no 241 dramatic increase in PHB was observed for these knockouts. One possible explanation is that 242 PHB production was being limited by NADPH availability rather than peroxisomal acetyl-CoA.

243 Further testing is needed to ensure sufficient NADPH availability and to determine the

244 biosensor's full dynamic range.

246 Unlike the two-day cultures used for Fig. 2, the five-day cultures in the knockout experiments 247 showed low levels of PHB production even in the absence of oleic acid. This could be caused by 248 acetate being produced and converted to acetyl-CoA in the peroxisome by the pyruvate 249 dehydrogenase bypass after the glucose has been depleted(Nielsen, 2014). PHB might also be 250 produced by low, native availability of acetoacetyl-CoA and 3-hydroxybutyric-CoA 251 intermediates. In a study by Leaf et al., yeast cells expressing only the final enzyme, PHB 
252 synthase, were able to produce PHB, albeit at only $1 \mathrm{mg} / \mathrm{g}$ DCW(Leaf et al., 1996). Intermediates

253 produced in this manner would still be positively correlated to acetyl-CoA availability in the

254 peroxisome, and thus, do not interfere with the intended function of the biosensor.

255

256

\section{Conclusions}

258

259 In summary, we developed an assay that used three compartmentalized enzymes to convert 260 peroxisomal acetyl-CoA to a reporter compound, PHB. By quantifying PHB levels, we

261 successfully applied this compartmentalized biosensor to screen a library of yeast mutants and 262 identified knockouts with drastically impaired PHB synthesis, which suggests the possibility that 263 overexpression of some of those genes might result in increased acetyl-CoA availability in the 264 peroxisome. Further testing is needed to ensure that PHB levels correlate quantifiably to acetyl265 CoA availability, that NADPH availability does not become limiting and to ensure proper 266 enzyme localization. Further testing is also needed to ensure that differences in PHB production 267 are not caused by differences in enzyme expression among the different strains and culturing 268 conditions used. Metabolic engineering of compartmentalized pathways requires measurement 269 tools for pathway design and evaluation. Traditional methods for measuring metabolite 270 concentrations are generally limited to cytosolic or whole-cell sources of the metabolite. In 271 contrast, as shown in this study, compartmentalized biosensors could be a valuable addition to 272 current synthetic toolkits on analyzing intracellular pools of metabolites.

273

274 This study illustrates only one specific application of a compartmentalized biosensor, but the 275 strategy could be applied to other metabolites in other compartments. For example, the 276 concentrations of acetyl-CoA in the mitochondria have been estimated to be 20-30 times higher 277 than in the cytosol(Weinert et al., 2017) and a recent study involved re-localizing heterologous 278 enzymes for the production of valencene to the mitochondria of S. cerevisiae via targeting signal 279 peptides resulting in increased production compared to cytosolic expression of those same 280 enzymes(Farhi et al., 2011). Further optimization of this localized pathway could benefit from a 281 biosensor similar to ours to detect the critical precursor, farnesyl diphosphate (FDP). FDP can be 282 detected using a fluorescent enzymatic assay(Dozier \& Distefano, 2012) and 283 compartmentalization of this assay could be accomplished by introducing N-terminal 284 mitochondrial localization signal (MLS) tags such as from subunit IV of the yeast cytochrome C 285 oxidase (CoxIV) to the enzymes of the fluorescent assay(Avalos, Fink \& Stephanopoulos, 2013).

286

287 We anticipate that compartmentalized biosensors can be used to better understand the

288 compartmentalized metabolism in various organelles and facilitate "design-build-test" cycle of 289 compartmentalized metabolic engineering.

290

291

292 
293 References

294

295

296

297

298

299

300

301

302

303

304

305

306

307

308

309

310

311

312

313

314

315

316

317

318

319

320

321

322

323

324

325

326

327

328

329

330

331

332

333
Avalos JL, Fink GR, Stephanopoulos G. 2013. Compartmentalization of metabolic pathways in yeast mitochondria improves the production of branched-chain alcohols. Nature Biotechnology 31:335-341. DOI: 10.1038/nbt.2509.

Blank LM, Sauer U. 2004. TCA cycle activity in Saccharomyces cerevisiae is a function of the environmentally determined specific growth and glucose uptake rates. Microbiology 150:1085-1093. DOI: 10.1099/mic.0.26845-0.

Brognaux A, Thonart P, Delvigne F, Neubauer P, Twizere J, Francis F, Gorret N. 2013. Direct and indirect use of GFP whole cell biosensors for the assessment of bioprocess performances: Design of milliliter scale down bioreactors. Biotechnology Progress 29:48-59. DOI: 10.1002/btpr.1660.

Chen Y, Siewers V, Nielsen J. 2012. Profiling of cytosolic and peroxisomal acetyl-CoA metabolism in Saccharomyces cerevisiae. PLoS One 7:e42475. DOI: 10.1371/journal.pone.0042475.

Choi H-J, Montemagno CD. 2006. Biosynthesis within a bubble architecture. Nanotechnology 17. DOI: $10.1088 / 0957-4484 / 17 / 9 / 020$.

DeLoache WC, Russ ZN, Dueber JE. 2016. Towards repurposing the yeast peroxisome for compartmentalizing heterologous metabolic pathways. Nat Commun 7:11152. DOI: 10.1038/ncomms11152.

Dozier JK, Distefano MD. 2012. An enzyme-coupled continuous fluorescence assay for farnesyl diphosphate synthases. Anal Biochem 421:158-63. DOI: 10.1016/j.ab.2011.10.038.

Farhi M, Marhevka E, Masci T, Marcos E, Eyal Y, Ovadis M, Abeliovich H, Vainstein A. 2011. Harnessing yeast subcellular compartments for the production of plant terpenoids. Metabolic Engineering 13:474-481. DOI: 10.1016/j.ymben.2011.05.001.

Fritz V, Benfodda Z, Henriquet C, Hure S, Cristol JP, Michel F, Carbonneau MA, Casas F, Fajas L. 2013. Metabolic intervention on lipid synthesis converging pathways abrogates prostate cancer growth. Oncogene 32:5101-10. DOI: 10.1038/onc.2012.523.

Guo W, Chen Y, Wei N, Feng X. 2016. Investigate the Metabolic Reprogramming of Saccharomyces cerevisiae for Enhanced Resistance to Mixed Fermentation Inhibitors via 13C Metabolic Flux Analysis. PLoS One 11:e0161448. DOI: 10.1371/journal.pone.0161448.

Gurvitz A, Rottensteiner H. 2006. The biochemistry of oleate induction: transcriptional upregulation and peroxisome proliferation. Biochim Biophys Acta 1763:1392-402. DOI: 10.1016/j.bbamcr.2006.07.011.

Jose S, Suraishkumar GK. 2016. High carbon (CO2) supply leads to elevated intracellular acetyl CoA levels and increased lipid accumulation in Chlorella vulgaris. Algal Research 19:307-315. DOI: 10.1016/j.algal.2016.08.011.

Joska TM, Mashruwala A, Boyd JM, Belden WJ. 2014. A universal cloning method based on yeast homologous recombination that is simple, efficient, and versatile. J Microbiol Methods 100:46-51. DOI: 10.1016/j.mimet.2013.11.013. 
334 Karr DB, Waters JK, Emerich DW. 1983. Analysis of Poly- $\beta$-Hydroxybutyrate in Rhizobium

335

336

337

338

339

340

341

342

343

344

345

346

347

348

349

350

351

352

353

354

355

356

357

358

359

360

361

362

363

364

365

366

367

368

369

370

371

372

373

374

375 japonicum Bacteroids by Ion-Exclusion High-Pressure Liquid Chromatography and UV Detection †. Appl Environ Microbiol 46:1339-44.

Kikuchi M, Hatano N, Yokota S, Shimozawa N, Imanaka T, Taniguchi H. 2004. Proteomic analysis of rat liver peroxisome: presence of peroxisome-specific isozyme of Lon protease. J Biol Chem 279:421-8. DOI: 10.1074/jbc.M305623200.

Kim PK, Hettema EH. 2015. Multiple pathways for protein transport to peroxisomes. J Mol Biol 427:1176-90. DOI: 10.1016/j.jmb.2015.02.005.

Kocharin K, Chen Y, Siewers V, Nielsen J. 2012. Engineering of acetyl-CoA metabolism for the improved production of polyhydroxybutyrate in Saccharomyces cerevisiae. AMB Express 2:52. DOI: 10.1186/2191-0855-2-52.

Leaf TA, Peterson MS, Stoup SK, Somers D, Srienc F. 1996. Saccharomyces cerevisiae expressing bacterial polyhydroxybutyrate synthase produces poly-3-hydroxybutyrate. Microbiology 142 ( Pt 5):1169-80. DOI: 10.1099/13500872-142-5-1169.

Li Y, Yu Z. 2015. Construction and evaluation of a genetic construct for specific detection and measurement of propionate by whole cell bacteria. Biotechnology and Bioengineering 112:280-287. DOI: 10.1002/bit.25358.

Liu H, Naismith JH. 2008. An efficient one-step site-directed deletion, insertion, single and multiple-site plasmid mutagenesis protocol. In: BMC Biotechnol. 91. DOI: 10.1186/14726750-8-91.

Mahr R, von Boeselager RF, Wiechert J, Frunzke J. 2016. Screening of an Escherichia coli promoter library for a phenylalanine biosensor. Applied Microbiology and Biotechnology 100:6739-6753. DOI: 10.1007/s00253-016-7575-8.

Michener JK, Thodey K, Liang JC, Smolke CD. 2012. Applications of genetically-encoded biosensors for the construction and control of biosynthetic pathways. Metabolic Engineering 14:212-222. DOI: 10.1016/j.ymben.2011.09.004.

Mustafi N, Grünberger A, Kohlheyer D, Bott M, Frunzke J. 2012. The development and application of a single-cell biosensor for the detection of l-methionine and branched-chain amino acids. Metabolic engineering 14:449-457. DOI: 10.1016/j.ymben.2012.02.002.

Ngo TT, Lenhoff HM. 1980. A sensitive and versatile chromogenic assay for peroxidase and peroxidase-coupled reactions. Anal Biochem 105:389-97.

Nielsen J. 2014. Synthetic Biology for Engineering Acetyl Coenzyme A Metabolism in Yeast. In: mBio. DOI: 10.1128/mBio.02153-14.

Shao Z, Luo Y, Zhao H. 2011. Rapid characterization and engineering of natural product biosynthetic pathways via DNA assembler. Mol Biosyst 7:1056-9. DOI: 10.1039/c0mb00338g.

Shao Z, Zhao H. 2009. DNA assembler, an in vivo genetic method for rapid construction of biochemical pathways. Nucleic Acids Res 37:e16. DOI: 10.1093/nar/gkn991.

Skjoedt ML, Snoek T, Kildegaard KR, Arsovska D, Eichenberger M, Goedecke TJ, Rajkumar AS, Zhang J, Kristensen M, Lehka BJ, Siedler S, Borodina I, Jensen MK, Keasling JD. 2016. Engineering prokaryotic transcriptional activators as metabolite biosensors in yeast. Nature Chemical Biology 12:951-958. DOI: 10.1038/nchembio.2177. 
376 Wang J, Yu HQ. 2007. Biosynthesis of polyhydroxybutyrate (PHB) and extracellular polymeric

377

378

379

380

381

382

383

384

385

386

387

388

389

390

391

392

393

394 substances (EPS) by Ralstonia eutropha ATCC 17699 in batch cultures. Appl Microbiol Biotechnol 75:871-8. DOI: 10.1007/s00253-007-0870-7.

Weinert BT, Denmark TNC for PRF of HSU of CC, Iesmantavicius V, Denmark TNC for PRF of HSU of CC, Moustafa T, Austria I of MBU of GG, Schölz C, Denmark TNC for PRF of HSU of CC, Wagner SA, Denmark TNC for PRF of HSU of CC, Magnes C, Austria H- I for B and HSJRG, Zechner R, Austria I of MBU of GG, Choudhary C, Denmark TNC for PRF of HSU of CC. 2017. Acetylation dynamics and stoichiometry in Saccharomyces cerevisiae. Molecular Systems Biology 10. DOI: 10.1002/msb.134766.

Zecchin A, Stapor PC, Goveia J, Carmeleit P. 2015. Metabolic pathway compartmentalization: an underappreciated opportunity? Current Opinion in Biotechnology 34:73-81. DOI: 10.1016/j.copbio.2014.11.022.

Zhou YJ, Buijs NA, Zhu Z, Gomez DO, Boonsombuti A, Siewers V, Nielsen J. 2016. Harnessing Yeast Peroxisomes for Biosynthesis of Fatty-Acid-Derived Biofuels and Chemicals with Relieved Side-Pathway Competition. J Am Chem Soc 138:15368-15377. DOI: 10.1021/jacs.6b07394. 
Figure 1

Mechanism of the localized biosensor.

The three PHB enzymes were expressed from a plasmid and contained enhanced PTS1 localization tags. The tags were recognized by peroxisomal targeting signal receptor Pex5p which shuttles the enzymes into the peroxisome. There, the enzymes converted peroxisomal acetyl-CoA into PHB that was then extracted and detected by HPLC.

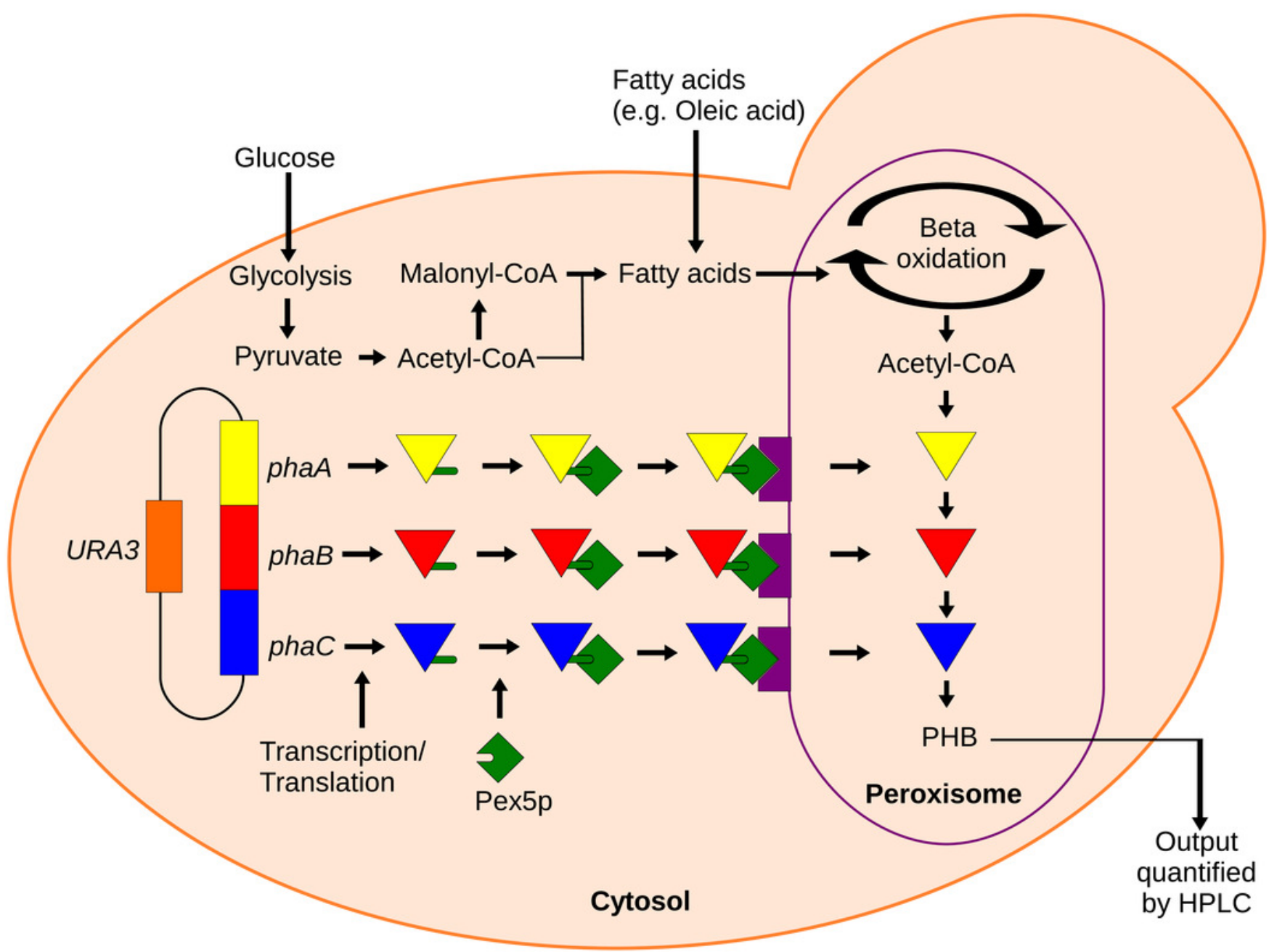




\section{Figure 2}

Oleic acid dependency of peroxisomal PHB.

Yeast strains with PHB producing enzymes were incubated for 48 hours in several media and with varied concentrations of oleic acid. A) Yeast strain phbC containing the three pha enzymes without enzyme localization. B) Yeast strain phbP with the three pha enzymes with localization tags. ND: Not Detectable. Error bars show estimated instrument error.
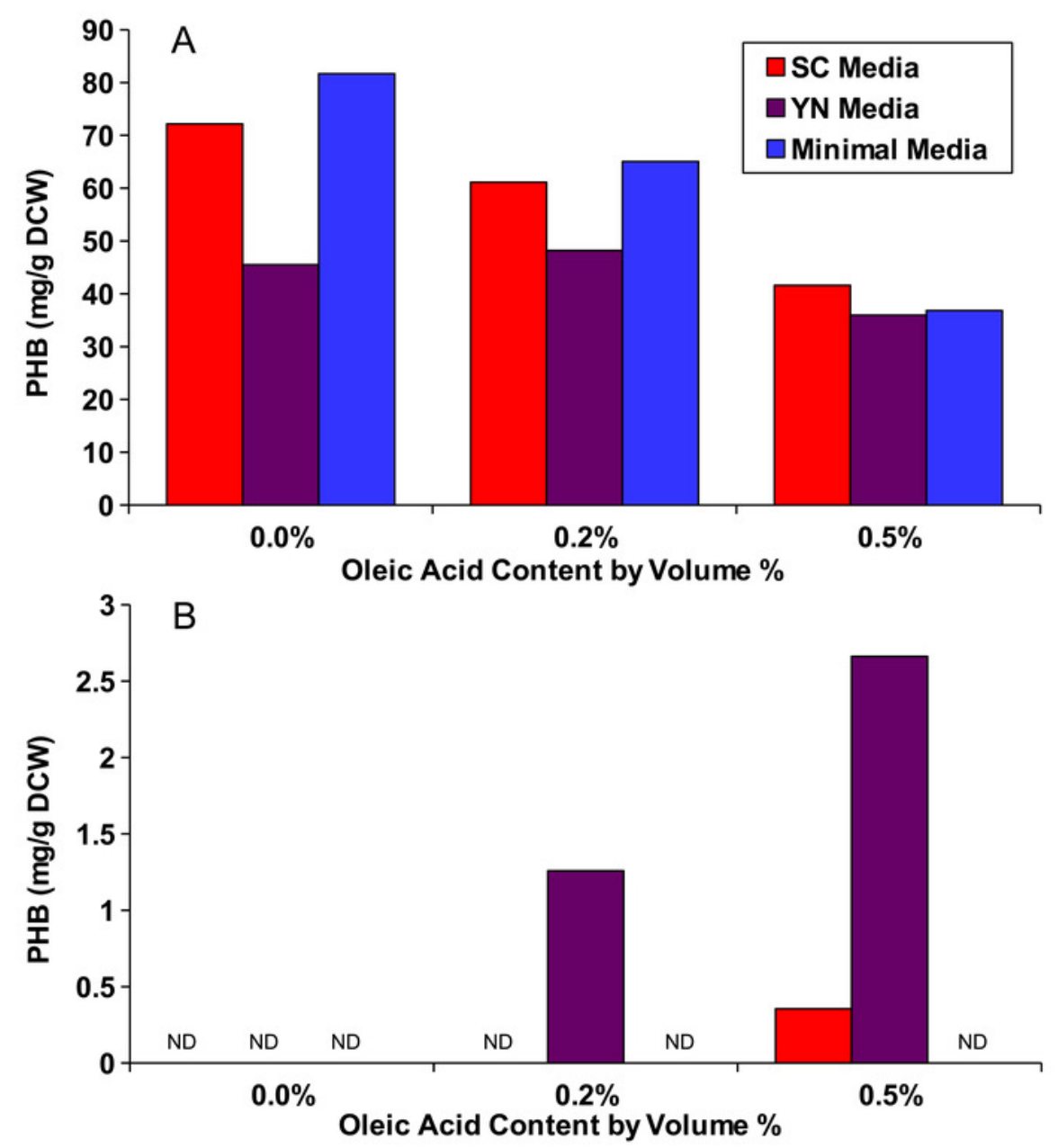
Figure 3

PHB production and growth curves for cytosolic and peroxisomal strains.

Both strains were cultured for five days in YN media with $0.5 \%$ oleic acid. A) PHB content vs. time for the cytosolic strain phbC. B) PHB content vs. time for phbP. C) Growth curve for the phbC. D) Growth curve for phbP. Error bars show standard deviation among triplicate cultures.
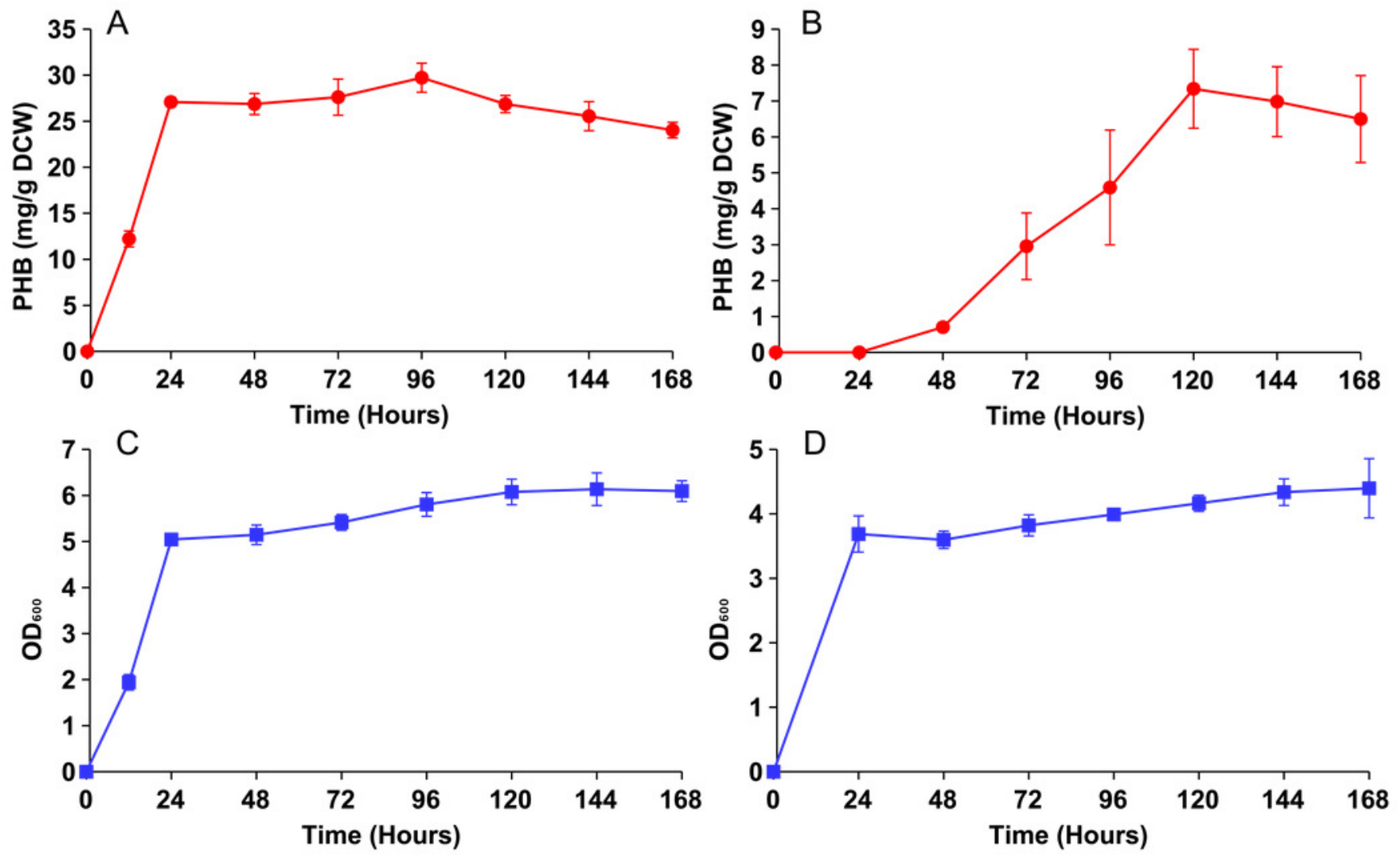


\section{Figure 4}

Figure 4. Screening of mutants using the peroxisomal acetyl-CoA assay.

Yeast knockout strains and the wild type (WT) were incubated for five days in YN media with various oleic acid concentrations. A) PHB concentration. B) Cell density (in $\mathrm{OD}_{600}$ ) of the mutant screening cultures. ND: Not Detectable. Error bars show estimated instrument error.
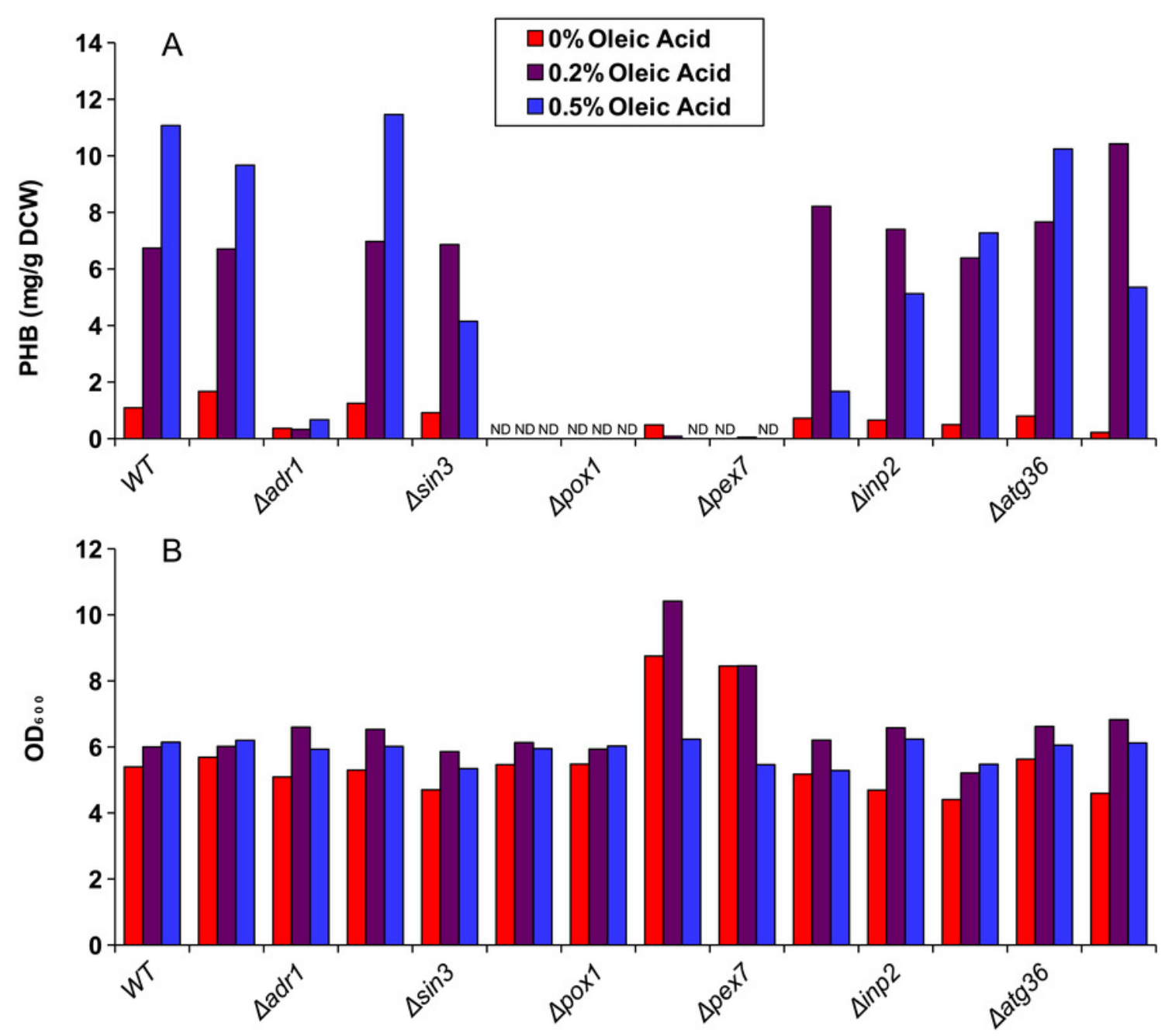


\section{Table $\mathbf{1}$ (on next page)}

Plasmids and strains used in this study.

Plasmids and strains ending in the letter " $\mathrm{C}$ " for "cytosolic"express heterologous PHB enzymes without localization tags. Plasmids and strains ending in " $\mathrm{p}$ " for "peroxisomal" express enzymes modified with enhanced Peroxisome Targeting Sequences (ePTS1). 
1

\begin{tabular}{lll}
\hline $\begin{array}{l}\text { Plasmid } \\
\text { Name }\end{array}$ & Description & \\
\hline pPHBc & $\begin{array}{l}\text { pRS416-(adh1t-phaA-pgk1p)-(tef1p-phaB-cyc1t)-(pdc1p-phaC- } \\
\text { pdc1t) } \\
\text { pRS416-(adh1t-ePTS1-phaA-pgk1p)-(tef1p-phaB-ePTS1-cyc1t)- } \\
\text { (pdc1p-phaC-ePTS1 pdc1t) }\end{array}$ & \\
\hline Strains & & Plasmid \\
Name & Phenotype & pPHBc \\
\hline BY4741 & MATa his3 $\Delta 1$ leu2 $\Delta 0$ met15 $\Delta 0$ ura3 $\Delta 0$ & pPHBp \\
phbC & Same as BY4741 & \\
\hline
\end{tabular}

2 